\title{
The Influence of Inlet Pressure Control on Separation Performance of Multi-Product Cyclones
}

\author{
Tingrui Liu ${ }^{*}$, Peikun Liu, Zengyin Wang and Ziqing Yu \\ College of Mechanical \& Electronic Engineering, Shandong University of Science \& Technology, Qingdao, China
}

\begin{abstract}
This paper is devoted and intended to solve the problems in determining the precise separation efficiency and accurate prediction for the solid yield of multi-product cyclones based on existing experimental data. The influence of inlet pressure control on separation performance of multi-product cyclones is investigated. Hydrocyclone separation performance is influenced by many factors such as the liquid level of agitating vessel and the entrance pressure. The liquid level can also be controlled through the entrance pressure control. The mathematical model of multi-product cyclone system is a high-order complex model and it is difficult to determine the specific expressions. The paper adopts a special optimal fuzzy PI_PID control strategy performed by Programmable Logic Controller system to enable inlet pressure control. By the force of contrast with experiment and analysis for many performance indexes, the effectiveness and applicability of the control approach are demonstrated. The research provides a method for control of high-order complex system of hydrocyclone separation.
\end{abstract}

Keywords: Inlet pressure, Multi-product cyclones, Optimal fuzzy PI_PID control strategy, Programmable logic controller system, Separation performance.

\section{INTRODUCTION}

During the existing cyclone separation production process, liquid level fluctuation of pump pool (agitating vessel) has great influence on separation efficiency [1]. If the pulp level is too high, it is easy to cause the slurry overflow loss. Conversely, if the pulp level is too low, it is easy for the air to enter the pump, resulting in cavitation, directly affecting the pump output, and then affects the hydraulic stability in hydrocyclone, and reduces the classification effect of hydrocyclone. When the hydraulic pressure of hydrocyclone is lower than the stable value, very fine slurry cannot be separated and it will cause a pulp overflow. Therefore it is more crucial in multi-product cyclone system to ensure the height of the liquid level of agitating vessel is established [2-6].

In this paper, the liquid level can be controlled through the entrance pressure control by specific control system and variable pump system. At the same time, the stability of the import pressure itself, can also guarantee high efficiency and stability of separation performance, which is demonstrated by comparing with the obtained experimental results. The experimental system is illustrated in Fig. (1). The system includes two cyclones with different sizes (cyclone I: $\varphi 50$ $\mathrm{mm}$; cyclone II: $\varphi 25 \mathrm{~mm}$;), four sampling recycling bins, agitating vessel, variable pump, transducer, Programmable Logic Controller (PLC) system and touch screen, etc. The work procedure is as follows:

*Address correspondence to this author at the College of Mechanical \& Electronic Engineering, Shandong University of Science \& Technology, No. 579, Qianwangang Road, Qingdao, Postcard: 266590, China;

Tel: 15689977136; E-mail: liutingrui9999@163.com
The slurry is classified through two cyclones, from the underflow and overflow, returns to the stirred vessel, and goes into a circulating form. Two pressure gauges are presented, and the manual valve opening degree and variable pump are adjusted. Preliminarily the inlet pressures of the two cyclones is set up. The entrance of cyclone is provided with a pressure sensor, and the pressure controlled by the PLC control system. When the pressure is stabilized, from the underflow and overflow, synchronous sampling is performed.

\section{CONTROL STRATEGY}

\subsection{Preliminary Estimation and Identification of System Model}

In order to evaluate and control such a complex system, a mathematical model should be preliminarily estimated by the least squares algorithm based on the method of minimized sum of squared residuals (LSR)[7]. By LSR method, the original system can be excited by the input signal sequence (inlet pressure signals) to generate the output signal sequence (transducer frequency signals). Based on these signals, the discrete-time model can be identified. The discrete-time model (discrete transfer function model) converted from the identified results is actually a double input transfer function matrix. It includes the expected transfer function model and the transfer function from error signal to the output signal (should be discarded in this example) [7]. So the five-order continuous transfer function model (converted from the discrete transfer function model) of the identified multiproduct cyclones system can be obtained as: 


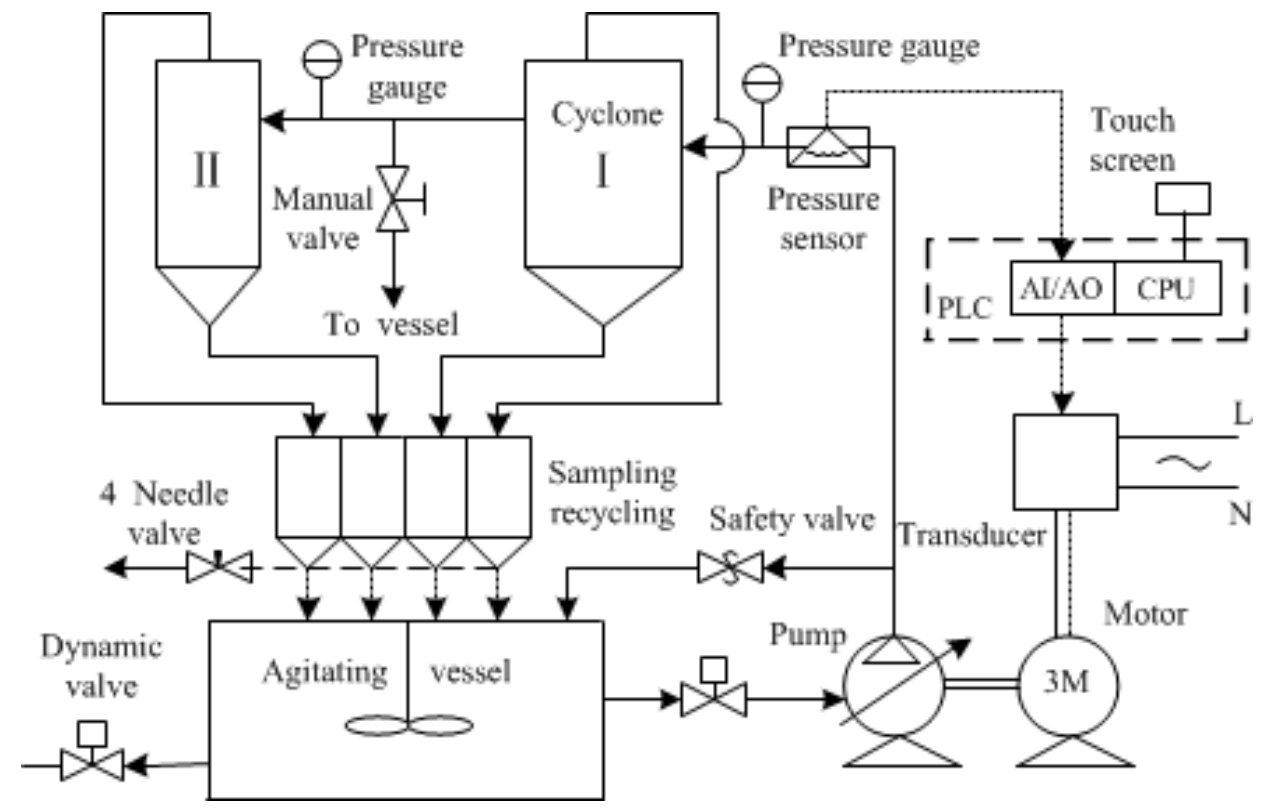

Fig. (1). The experimental system.

$$
G=e^{-B s} \frac{A}{a_{5} s^{5}+a_{4} s^{4}+a_{3} s^{3}+a_{2} s^{2}+a_{1} s+a_{0}}
$$

Unfortunately, the related parameters $a_{\mathrm{i}}, A$, and $B$ in $G$ are not fixed, but vary during different testing processes. It might be caused by system complexity, system internal dynamic fluctuation and outside interference, and/or very high nonlinearity of dynamics. The nonlinear theory for this cyclone system has far surpassed what the above proposed model can display. Therefore, it is difficult to control inlet pressure stability by conventional methods for such an unknown system.

\subsection{Optimal Fuzzy_PI PID Control Strategy}

In recent years, some advanced control strategy including fuzzy control and neural network control such as Back Propagation Neural Network (BPNN), Radial Basic Functions Neural Network (RBFNN) and Generalized Regression Neural Network (GRNN), are employed to control and identify related process of cyclone system. References [1-2] utilize fuzzy control to control the pulp level of the classification pump-sump in metal mineral processing. It has solved the problem of the variation of pump throughput due to the sharp variation of the pumpsump pulp level that leads to a reduction of the hydrocyclone classification efficiency. References [3-4] take the heavy medium hydrocyclone as an object of control, and introduce fuzzy control and neural network to construct heavy medium hydrocyclone intelligent control system based on the traditional PID control. A fuzzy PID control algorithm is used to control the inlet pressure by variable frequency speed governing technology to solve the problem of low classification efficiency due to the instable pressure of hydrocyclone [4]. Reference [8] uses a three-layer BPNN model capable of predicting separated particle size, production capacity, and the underflow concentration, and obtains comprehensive prediction of the separator performance indexes under special structure and the designed parameters. For the purpose of building the complex relationships between cyclone pressure drop coefficient and geometrical dimensions, representative artificial neural networks, including BPNN, RBFNN and GRNN, are developed to model pressure drop coefficient for cyclone separators [9]. For the purpose of accurate prediction of the complex nonlinear relationships between performance parameters and the geometrical dimensions, two types of RBFNN algorithms are investigated and employed to model the pressure drop and the cut-off diameter for cyclone separators [10]. However, all these intelligent algorithms are concentrated in the application of single cyclone system. In the application of this paper, it is found that all of the above algorithms cannot achieve satisfactory results. So in order to realize such an unknown high-order complex system, a special optimal fuzzy_PI PID control strategy is investigated here and satisfactory results are obtained.

In view of the fact that fuzzy control depends on the fuzzy algorithm between the information of process and control input, fuzzy controller has demonstrated a large range of applicability to processes where the transfer functions are not precisely defined but the control actions can be described in terms of linguistic variables [11-13]. PID controller is still the most effective controller for process control applications. PID controller parameters can be tuned with three variables: proportional gain $(\mathrm{Kp})$, integral gain $\left(\mathrm{K}_{\mathrm{I}}\right)$ and derivative gain $(\mathrm{Kd})$. For this system with unknown parameters, an optimal fuzzy_PI PID controller is devised. Its main advantage lies in that the controller parameters can be changed quickly and optimized in response to changes in system dynamics. Fig. (2) shows the block diagram of fuzzy_PI PID control strategy. Two input variables, error $(E)$ and change of error $(C E)$ and two output variables $\mathrm{Kp}$ and $\mathrm{Ki}$ with 7 linguistic variables of membership function are used. The system knowledge base comprises fuzzy IF-THEN rules and membership functions. The input linguistic variables are negative large $(N L)$, negative medium $(N M)$, negative small $(N S)$, zero $(Z E)$, positive small $(P S)$, positive medium $(P M)$ and positive large $(P L)$. Seven triangular-membership 


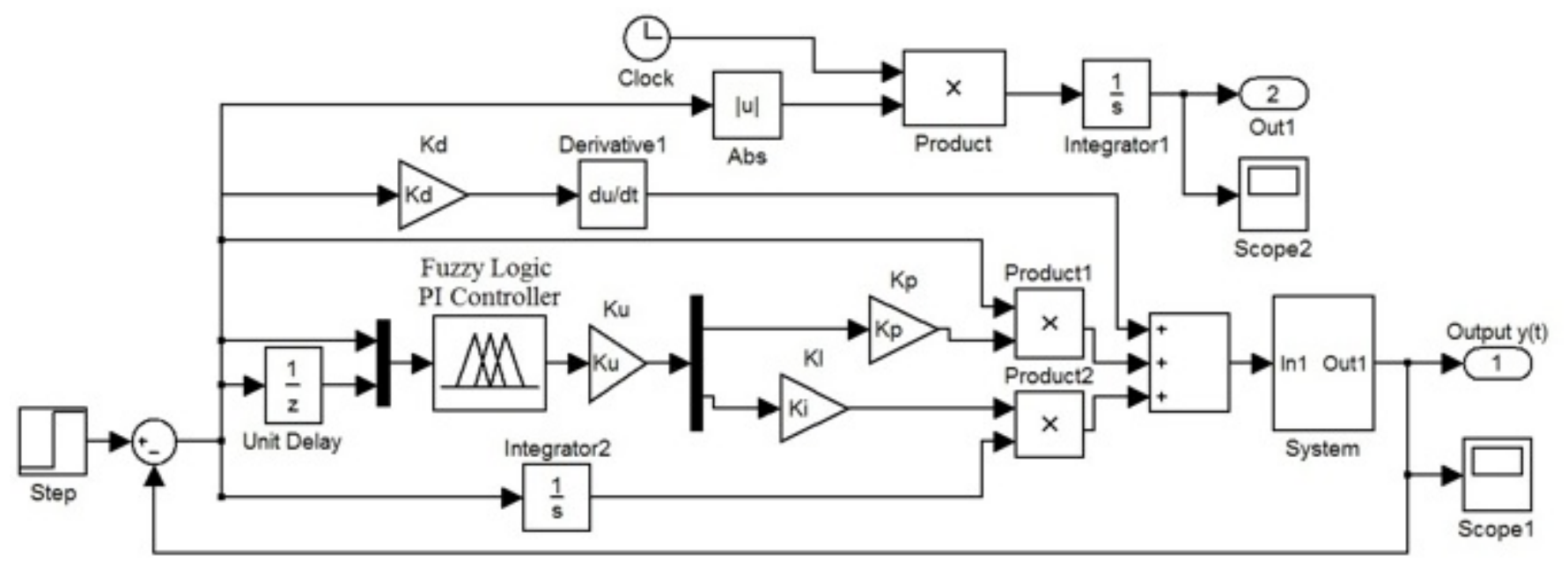

Fig. (2). Block diagram of optimal fuzzy_PI PID control strategy.

function forms for both $\mathrm{E}$ and $\mathrm{CE}$ are determined, which are automatically generated in Fuzzy Control Toolbox in MATLAB and shown in Fig. (3), where $m f 6$ means $N L$, $m f 1 \sim m f 5$ mean $N M, N S, Z E, P S$, and $P M$, respectively, and $\mathrm{mf} 7$ means $P L$. Borders of both function sequences vary between \pm 6 . In this study, a fuzzy rule type of the designed fuzzy_PI PID controller is developed based on Sugeno rules. So there are 49 weight values in both $\mathrm{Kp}$ and $\mathrm{K}_{\mathrm{I}}$ functions. According to intuition method, list of linguistic rules are shown in Tables $\mathbf{1}$ and $\mathbf{2}$. The aggregation and defuzzification methods are max-min and centroid approaches, respectively.

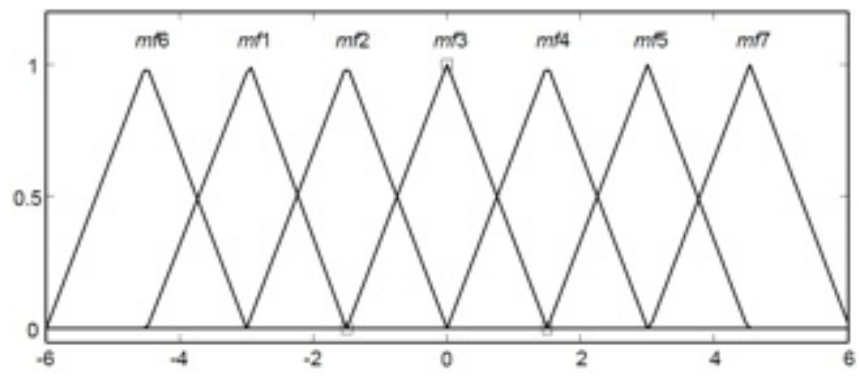

Fig. (3). Seven triangular-membership function forms for both $\mathrm{E}$ and CE.

Table 1. Rules of Kp.

\begin{tabular}{|c|c|c|c|c|c|c|c|}
\hline E $\backslash$ CE & NL & NM & NS & ZE & PS & PM & PL \\
\hline \hline NL & 1 & 1 & 0.85 & 0.85 & 0.65 & 0.5 & 0.5 \\
\hline NM & 1 & 1 & 0.85 & 0.65 & 0.5 & 0.5 & 0.35 \\
\hline NS & 0.85 & 0.85 & 0.85 & 0.65 & 0.5 & 0.5 & 0.35 \\
\hline ZE & 0.85 & 0.85 & 0.65 & 0.5 & 0.35 & 0.15 & 0.15 \\
\hline PS & 0.65 & 0.65 & 0.5 & 0.35 & 0.35 & 0.15 & 0.15 \\
\hline PM & 0.85 & 0.65 & 0.5 & 0.35 & 0.15 & 0.15 & 0 \\
\hline PL & 0.5 & 0.35 & 0.15 & 0.15 & 0.15 & 0 & 0 \\
\hline
\end{tabular}

Table 2. Rules of $K_{\mathrm{I}}$.

\begin{tabular}{|c|c|c|c|c|c|c|c|}
\hline E\CE & NL & NM & NS & ZE & PS & PM & PL \\
\hline \hline NL & 0 & 0 & 0.5 & 0.5 & 1 & 1.5 & 1.5 \\
\hline NM & 0 & 0 & 0.5 & 1 & 1 & 1.5 & 1.5 \\
\hline NS & 0 & 0.5 & 1 & 1 & 1.5 & 2 & 2 \\
\hline ZE & 0.5 & 0.5 & 1 & 1.5 & 2 & 2.5 & 2.5 \\
\hline PS & 0.5 & 1 & 1.5 & 2 & 2 & 2.5 & 3 \\
\hline PM & 1.5 & 1.5 & 2 & 2 & 2.5 & 3 & 3 \\
\hline PL & 1.5 & 1.5 & 2 & 2.5 & 2.5 & 3 & 3 \\
\hline
\end{tabular}

In addition, PID parameters, $\mathrm{Kp}, \mathrm{K}_{\mathrm{I}}, \mathrm{Kd}$, and fuzzy gain $\mathrm{Ku}$ are optimized by optimal control (see Fig. 2), which is under certain concrete conditions, aiming as special control object, and to make selected target maximal or least. The target function used here adopts ITAE index as follows [7]:

$$
J_{\text {ITAE }}=\int_{0}^{\infty} t|e(t)| d t
$$

It can be seen from the practice that the ITAE rules in MATLAB/Simulink are quite versatile in finding the optimal controllers. However, in some applications, it may not find a solution due to the poorly posed problem or because a good initial search point has not been found. This can be a drawback in conventional optimization algorithms, but many such problems can be avoided by setting parameter in Abs block (in Simulink) to saturate on integer overflow and performing multiple continuous optimization processes, or by intelligent use of genetic algorithm based on an understanding of the system behavior [14]. It should be stated that when the optimization is finished, the parameter values that are obtained can be directly used as a substitute for set of Kp, KI, Kd, and Ku in Fig. (2), respectively. So the optimization process is performed only in the first testing. The parameter values obtained can be calibrated into PLC to act as constant parameters. 
Control process is realized by PLC system (see Fig. 1). The hardware of the PLC system includes CPU module, analog I/O module, and digital I/O modules, etc. Analog input module reads signal through the pressure sensor, and transfers it into CPU module to process the data including fuzzy PID operation. Analog input module can export resulting signal to drive motor by transducer. Pressure data can be recorded and displayed in touch screen. With the change of frequency of transducer, the variable pump input power changes, which leads to changes in the output flow of variable pump, and realizes inlet pressure adjustment. The multi-product cyclones and PLC system are illustrated in Fig. (4) respectively.

(a)

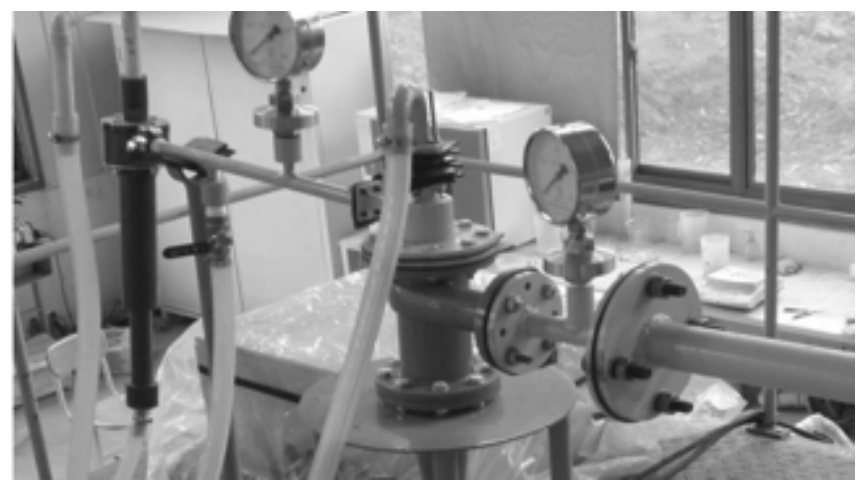

(b)

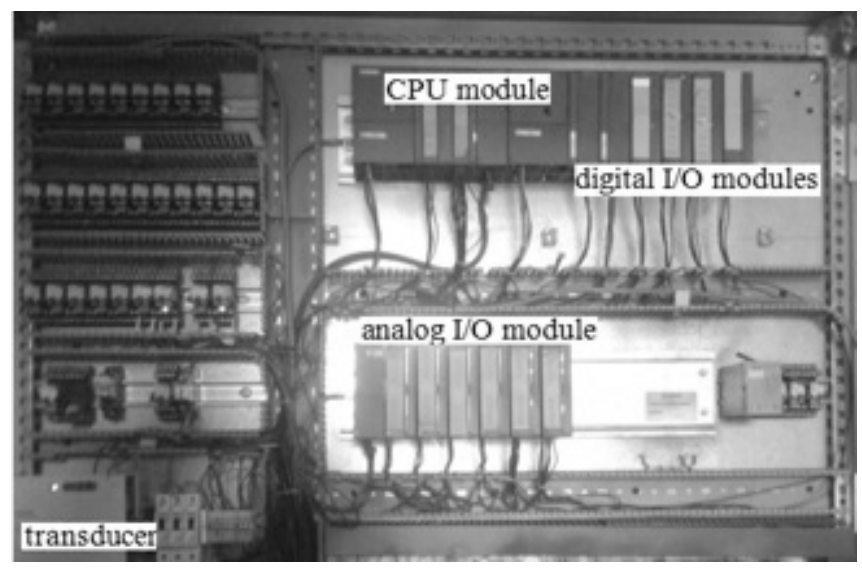

Fig. (4). The multi-product cyclones (a) and PLC system (b).

\section{RESULTS AND ANALYSIS}

\subsection{Validity of Control Methodology}

To testify the validity of the control methodology of optimal fuzzy_PI PID, fresh water testing sample is considered, the operating pressures are set at $0.10 \mathrm{MPa}, 0.13$ $\mathrm{MPa}, 0.15 \mathrm{MPa}$, and $0.18 \mathrm{MPa}$, respectively. Fig. (5a) shows different stable inlet pressures and their fluctuation processes. In general within $300 \mathrm{~s}$, the system can stabilize with small overshoot, which shows good performance regulation. Fig. (5b) shows touch screen and its curve display process. (a)

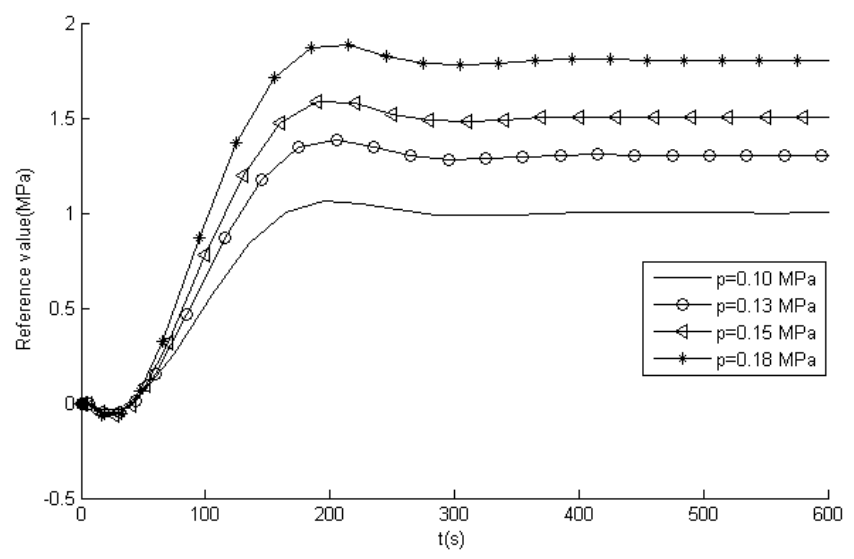

(b)

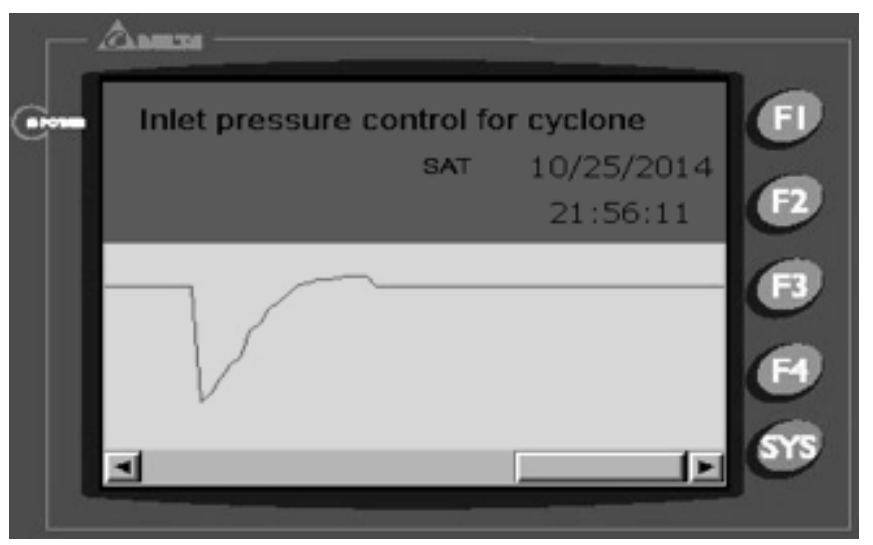

Fig. (5). Different inlet pressures and the fluctuation processes (a), and touch screen and its curve display (b).

\subsection{Influence of Pressure on Split Ratio}

Split ratio is the flux ratio of underflow and overflow. To investigate influence of pressure on split ratio, still considering fresh water testing sample, start the measuring process when the pressure goes into the stable region (five minutes later). Fig. (6) shows the change of split ratio against pressure from $0.12 \mathrm{Mp} \sim 0.20 \mathrm{Mp}$ an interval of $0.02 \mathrm{Mp}$. By comparison with the uncontrolled cases, whether the total split ratio or the second sectional split ratio (split ratio of cyclone II) changes smoothly and stably. At the same time, total split ratio changes little, while there is a large change for $2^{\text {nd }}$ sectional split ratio whether for controlled situation or not. Control process lays the foundation for accurate prediction of performance change for split ratio.

\subsection{Influence of Pressure on Separation Performance of Iron Tailings Materials}

The sampling materials are iron tailings with fine granularity with -200 mesh $(74 \mu \mathrm{m})$ up to $50.47 \%$ and -325 mesh $(44 \mu \mathrm{m})$ reaching $29.72 \%$. The density of tailings is 2.7 $\mathrm{g} / \mathrm{cm}^{3}$. The tailings composition is detected by Laser Particle 


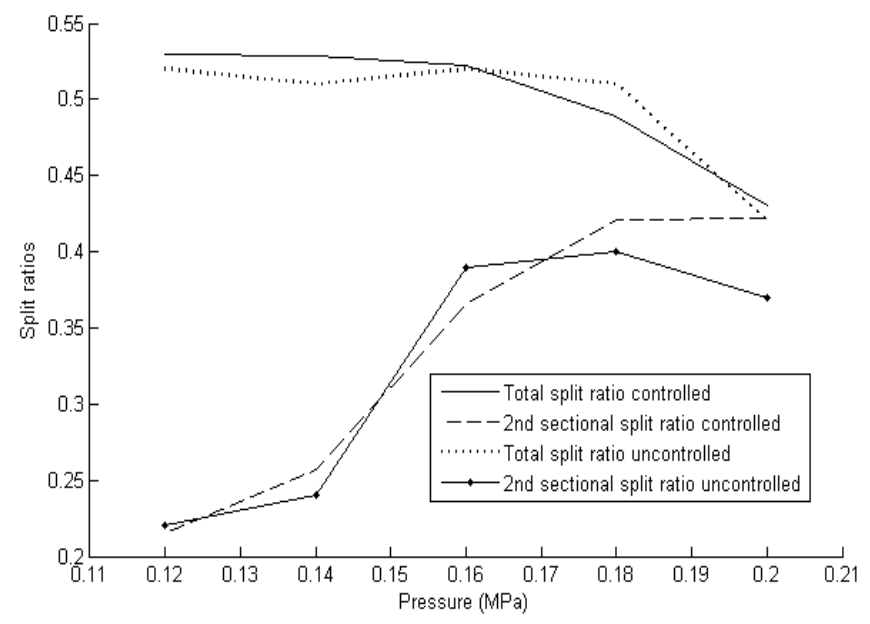

Fig. (6). Change of split ratio against pressure.

Analyzer (Type BT-9300S). Particle size distribution is illustrated in Fig. (7), with its specific report listed in reference [15].

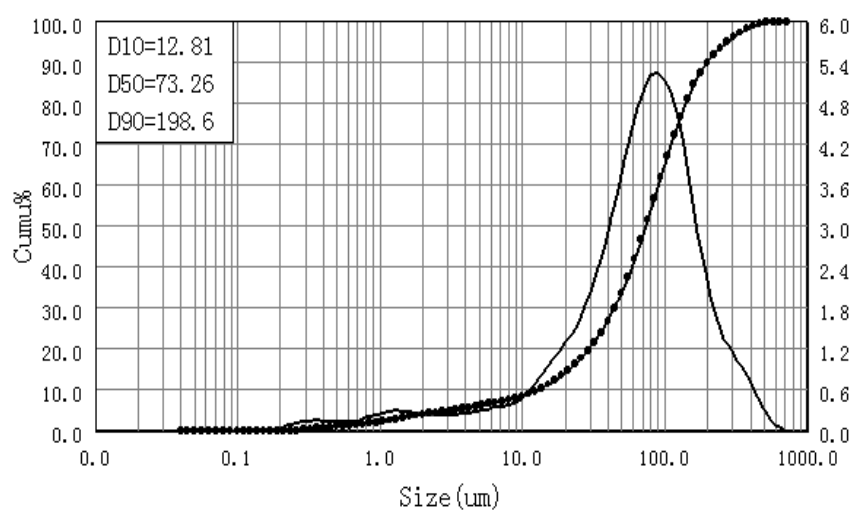

Fig. (7). Report of particle size distribution.

In the case of the median particle size of feeding tailings $75 \mu \mathrm{m}$, through the multi-product cyclones testing, four products received that have an average of the median particle size as: $1^{\text {st }}$ overflow (overflow in cyclonel) being $8 \mu \mathrm{m}, 1^{\text {st }}$ underflow being $95 \mu \mathrm{m}, 2^{\text {nd }}$ overflow (overflow in cyclonell) being $12 \mu \mathrm{m}$, and $2^{\text {nd }}$ underflow being $18 \mu \mathrm{m}$.
Fig. (8) shows the concentration change of overflow and underflow of two cyclones against pressure, respectively. By comparison with the overflow and underflow uncontrolled, the controlled cases tend to be stable, with more smooth curves. Its advantages lies in: avoiding impact of the system in the process of pressure fluctuations. On the other hand, it can also predict product yield variation with the pressure. Most importantly, the control process can precisely control the product yield.

Fig. (9) shows the solid yield of two cyclones and their classification efficiency ( -325 mesh for cyclonel, and -1250 mesh for cyclonell) against pressures. It can be seen that under a reasonable control strategy, the solid yield grows steadily, and the classification efficiency improves firmly. The solid yield of cyclonell and classification efficiency of cyclonel change, relatively greatly. The solid yield of cyclonel changes little with the increase of pressure. Compared with the controlled cases, the parameter values without control have greater fluctuation. The changing rules of controlled curves in turn, verify the validity of the control algorithm. So according to previous experimental values and pressure values, the late results can be predicted and accurately controlled with the increases of pressures.

\section{CONCLUSION}

The influence of inlet pressure control on separation performance indexes such as split ratio, concentration, solid yield, and classification efficiency, of multi-product cyclones is elaborately investigated. A special optimal fuzzy PI_PID control strategy is applied to realize in-let pressure control. The optimal control algorithm based on ITAE criterion is of significance in aspect of solutions for parametric objective functions, not only for PID controller parameter tuning, but also for fuzzy system parameter estimation, as described here. Hardware of control system is realized by PLC system with its corresponding attached modules. Under controlled situations, separation performance indexes change steadily with precise measuring values and relatively accurate prediction results. The proposed control strategy might lay a foundation for control of high-order complex multi-product cyclones system.
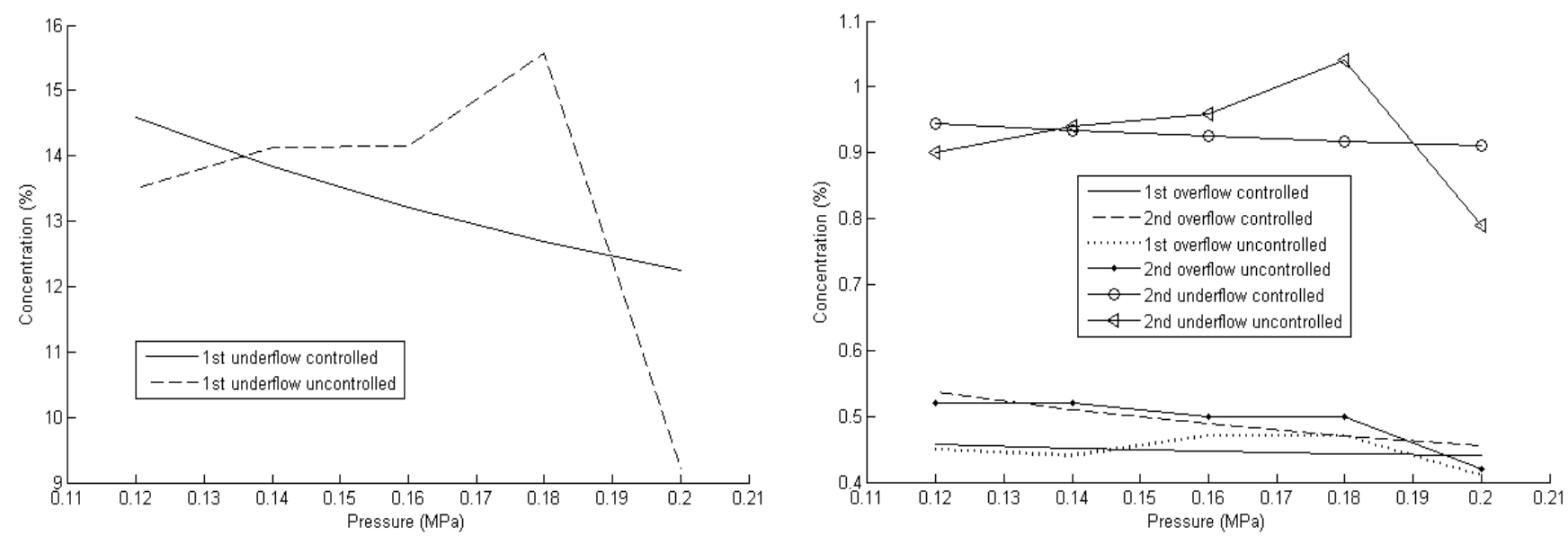

Fig. (8). Concentration change of overflow and underflow of two cyclones against pressure. 

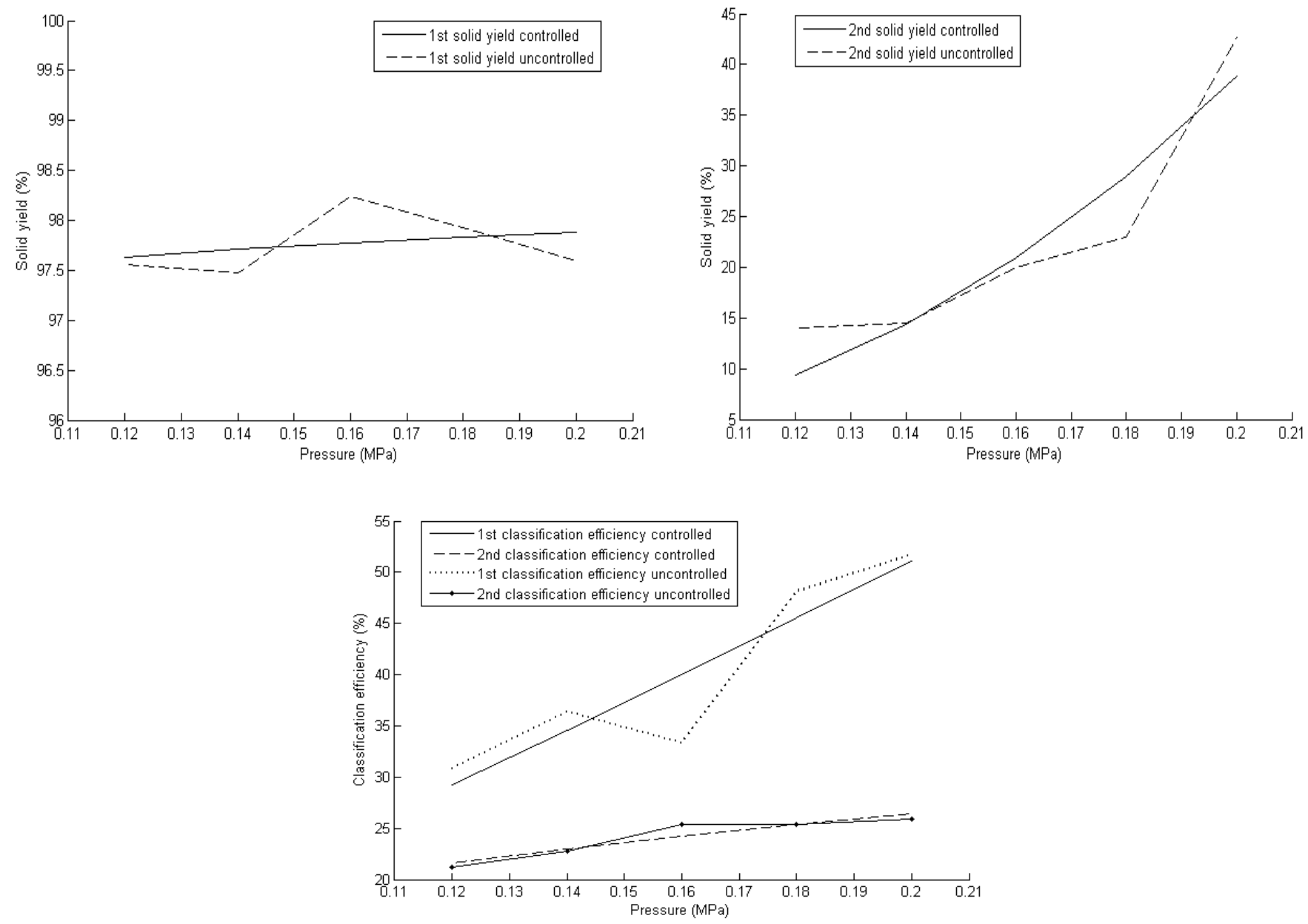

Fig. (9). The solid yield of two cyclones and their classification efficiency against pressures.

\section{CONFLICT OF INTEREST}

The authors declare that there is no conflict of interests regarding the publication of this article.

\section{ACKNOWLEDGEMENTS}

This work is supported by the National Natural Science Foundation of China (21276145), the Natural Science Foundation of Shandong Provincial of China (ZR2013AM016), and Qunxing Project of Shandong University of Science \& Technology (qx2013220).

\section{REFERENCES}

[1] D. Lu, J. Yu, and C. Guo, "Study on fuzzy control arithmetic for classification pump-sump pulp level in benefitiation", Metal Mine, vol. 9, no. 375, pp. 97-100, 2007. (In Chinese)

[2] L. Liang, and Z. Li, "Fuzzy intelligent control arithmetic for the ore pulp level in mineral separation process", Metal Mine, vol. 7, no. 397, pp. 104-105, 2009. (In Chinese)

[3] L. Ren, C. Lian, and J. Gao, "Density intelligent control of heavy medium hydrocyclone", Shanxi Coking Coal Science \& Technology, vol. 4, pp. 18-21, 2011. (In Chinese)

[4] X. Zhang, J. Yu, T. He, and C. Liu, "Fuzzy PID-based variable frequency speed constant pressure governing system for the classification in mineral process", Metal Mine, vol. 1, no. 391, pp.118-120, 2009. (In Chinese)

[5] J. Su, Z. Yuan, Y. Shi, and B. Chen, "Separation efficiency optimization of liquid-solid hydrocyclone and numerical simulation", Journal of Mechanical Engineering, vol. 47, no. 20, pp. 183-190, 2011. (In Chinese)

[6] B. Wang, and P. Cai, "Numerical simulation of multiphase flow field of non-newtonian fluid in hydrocyclone," CIESC Journal, vol. 63, no. 11 , pp. 3460-3469, 2012. (In Chinese)

[7] T. Liu, Y. Wang, and Q. Yang, "Optimal fuzzy control of electronic expansion valve-evaporator system", Bio Technology: An Indian Journal, vol. 8, no. 5, pp.586-594, 2013.

[8] L. Wei, C. Du, Y. Wang, and H. XU, "Prediction of artificial neural net-work-based hydrocyclones classification performance", Journal of Heilongjiang Institute of Science and Technology, vol. 22, no. 2, pp. 116-118, 2012. (In Chinese)

[9] Z. Bingtao, and S. Yaxin, "Artificial neural network-based modeling of pressure drop coefficient for cyclone separators", Chemical Engineering Research and Design, vol. 88, pp. 606-613, 2010.

[10] E. Khairy, and L. Chris, "Modeling and pareto optimization of gas cyclone separator performance using RBF type artificial neural networks and genetic algorithms", Powder Technology, vol. 217, pp. 84-89, 2012.

[11] O. Ekren, and S. Küüca, "Energy saving potential of a chiller system with fuzzy logic control", International Journal of Energy Research, vol.34, no.10, pp.897-906, 2010.

[12] E. Orhan, S. Savas, and I. Yalcin, "Comparison of different controllers for variable speed compressor and electronic expansion valve", International Journal of refrigeration, vol. 33, pp. 11611168, 2010.

[13] R. Dhanalakshmi, and S. Palaniswami, "Application of self-tuning fuzzy logic PI controller in load frequency control of wind-micro hydro-diesel hybrid power system", European Journal of Scientific Research, vol. 79, no. 3, pp. 317-327, 2012. 
[14] D. Y. Xue, and Y. Q. Chen, MATLAB Solutions to Mathematical Problems in Control, Beijing: Tsinghua University Publishing Company, 2007. (In Chinese)

[15] Z. Wang, Study on Separation Mechanism and Performance of Multi-Product Hydrocyclone, Ma. D. thesis, Shandong University of Science and Technology, Shandong, CN, May 29, 2014. (In Chinese)

Received: January 8, 2015

Revised: January 15, 2015

Accepted: January 16, 2015

(C) Liu et al.; Licensee Bentham Open.

This is an open access article licensed under the terms of the Creative Commons Attribution Non-Commercial License (http://creativecommons.org/licenses/by-nc/4.0/) which permits unrestricted, non-commercial use, distribution and reproduction in any medium, provided the work is properly cited. 